\title{
6.3 MeV Fast Neutrons in the Treatment of Patients with Locally Advanced and Locally Recurrent Breast Cancer
}

\author{
V. V. Velikaya ${ }^{1,2, a)}$, L. I. Musabaeva ${ }^{1, b)}$, V. A. Lisin ${ }^{1, c)}$, and Zh. A. Startseva $a^{1,2, d)}$ \\ ${ }^{I}$ Tomsk Cancer Research Institute, Kooperativny Street 5, Tomsk, 634050 Russia \\ ${ }^{2}$ National Research Tomsk Polytechnic University, Lenin Avenue 30, Tomsk, 634050 Russia

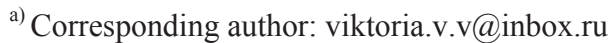 \\ b) musabaevaLI@oncology.tomsk.ru \\ c)Lisin@oncology.tomsk.ru \\ d) zhanna.alex@rambler.ru
}

\begin{abstract}
The study included 135 breast cancer patients (70 patients with locally recurrent breast cancer and 65 patients with locally advanced breast cancer with unfavorable prognostic factors) who received the neutron therapy alone or in combination with the photon therapy. The neutron therapy was shown to be effective in multimodality treatment of patients with locally advanced and locally recurrent breast cancer. The 8-year survival rate in patients without repeated breast cancer recurrence was $87.6 \pm 8.7 \%$ after the neutron and neutron-photon therapy and $54.3 \pm 9.2 \%$ after the electron beam therapy.
\end{abstract}

\section{INTRODUCTION}

A long-term experience in treating breast cancer has shown that the conventional postoperative radiation therapy including photon and electron therapy reduces the risk of breast cancer recurrence from $38 \%$ to $5 \%$ [1, 2]. The basic effect of ionizing radiation is to destroy the ability of cells to divide, by damaging their DNA strands. If a tumor cell is damaged by photon and electron radiation, it has a good chance to repair itself and continue to grow. By using neutron radiation therapy, the chance for a damaged tumor cell to repair itself is very small. Unlike photons, neutrons do not depend on the presence of oxygen to kill the cancer cells. In addition, the biological effectiveness of neutrons is not affected by the time or stage in the life cycle of cancer cells [3]. The application of $6.3 \mathrm{MeV}$ fast neutrons in treatment of malignant neoplasms is one of the possible ways to overcome radioresistance [4-6].

The purpose of the study was to evaluate the effectiveness of $6.3 \mathrm{MeV}$ fast neutrons in the treatment of patients with locally advanced and locally recurrent breast cancer.

\section{MATERIALS AND METHODS}

The therapeutic neutron bunch is received on a cyclotron U-120 when bombing a beryllium target by deuterons with energy of $13.5 \mathrm{MeV}$. The average energy of fast neutrons bunch is $\sim 6.3 \mathrm{MeV}$ [7-11].

A total of 227 breast cancer patients were included into the study. Eligibility criteria were as follows: locally advanced breast cancer recurrence; local recurrence after multimodality treatment of primary breast cancer with preoperative external beam radiotherapy delivered to the whole breast; radioresistant local recurrence of breast cancer after previously irradiated recurrent tumor; continuous tumor growth after multimodality treatment of infiltrative ductal breast carcinoma.

All patients were divided into 2 groups. Group I consisted of 114 patients with locally recurrent breast cancer $\left(\mathrm{T}_{2-4} \mathrm{~N}_{0-2} \mathrm{M}_{0}\right)$. This group was subdivided into the study group (70 patients) and the control group (44 patients). The study group patients received neutron therapy given twice a week with a 48-72 hour-interval using the U-120 cyclotron. 


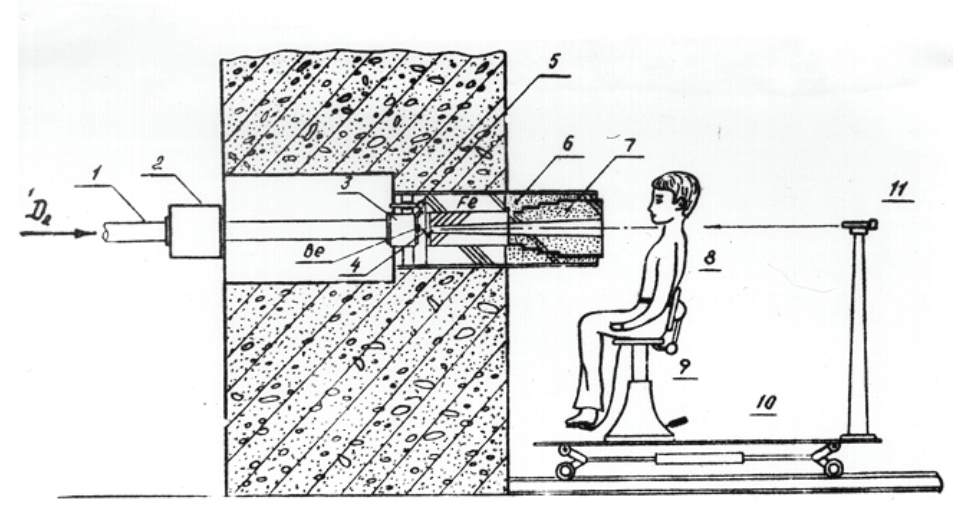

FIGURE 1. Therapeuticneutron beam scheme and the position ofthe patient duringirradiation session Radiation is carried out with the horizontal fixed bunch, at $110 \mathrm{~cm}$ distance from a target: 1,2-ionconductor, 3-beryllium target camera, 4-berylliumtarget, 5-iron shielding, 6-polyethylene shielding, 7-removable plastic collimator, 8-10 - parts of thetreatment chair, 11 -laser therapy device

The radiation field size was $96 \times 248 \mathrm{~cm}^{2}$ depending on the extent of local recurrence. Fast neutron beam was directed perpendicular to the chest wall at a source-to-skin surface distance of $110 \mathrm{~cm}$. Single radiation dose was 1.6-2.0 Gy (2.91-2.79 RBE), the skin dose was 2.0-2.2 Gy (2.79-2.73 RBE) [12-14]. Twenty-eight (37\%) of the 70 patients with locally advanced breast cancer recurrences received the neutron therapy alone at a total dose of 30 40 isoGy and $44(63 \%)$ patients received the combination of the neutron and photon therapy at a total dose of 5060 isoGy [15].

The control group included 44 patients with local breast cancer recurrence who received the electron beam therapy at a total dose of $60 \mathrm{~Gy}$. The mean follow-up time for 114 patients of both the study and the control groups was $7 \pm 0.5$ years.

Group II comprised 113 patients with stage $\mathrm{T}_{2-4} \mathrm{~N}_{0-3} \mathrm{M}_{0-1}$ locally advanced breast cancer with unfavorable prognostic factors, such as invasive ductal carcinoma, multicentric tumor growth and angiolymphaticinvansion. They underwent multimodality treatment including neoadjuvant chemotherapy (NACT) and adjuvant chemotherapy (ACT) according to CMF, CAF/FAC schemes, antiestrogen therapy (if indicated), radical mastectomy (RM) and radiotherapy.

The study group consisted of 65 breast cancer patients $\left(\mathrm{T}_{2-4} \mathrm{~N}_{0-3} \mathrm{M}_{0-1}\right)$, who from 2007 to 2014 received 34 sessions of the neutron therapy alone. The radiation field size was $96 \times 200 \mathrm{~cm}^{2}$. A single radiation dose was $1.4-$ 1.76 Gy with skin dose of 1.75-2.2 Gy. The total tumor dose was 16.7-29.9 isoGy with skin dose of 25.6-41 isoGy [16]. The control group comprised 48 breast cancer patients $\left(\mathrm{T}_{2-4} \mathrm{~N}_{0-3} \mathrm{M}_{0}\right)$ who, from 2004 to 2006, received electron beam therapy in a single dose of 3.0 Gy to the total dose of 38-44 isoGy delivered to the region of the anterior chest wall.

All 113 patients received the postoperative external beam radiotherapy at a total dose of 40-44 Gy delivered to the regional lymph nodes.

\section{RESULTS}

The neutron therapy was well tolerated and 1-2 grade radiation skin reactions were the most common.

In the group of patients with local breast cancer recurrence, 3 (4\%) out of the 70 patients had re-recurrences after previous neutron and neutron-photon therapy. In the control group after the electron beam therapy, repeated local recurrences of breast cancer were observed in $17(39 \%)$ of 44 patients. The 8-year survival rate in patients without repeated breast cancer recurrence was $87.6 \pm 8.7 \%$ after the neutron and neutron-photon therapy and $54.3 \pm 9.2 \%$ after the electron beam therapy $(p=0.0001)$.

Late radiation-induced damages to normal tissues. Twenty (16\%) out of 70 patients with local breast cancer recurrence after the neutron and neutron-photon therapy had late local radiation-induced skin and subcutaneous tissue injuries. When planning TDF, the analysis of the incidence of radiation-induced pulmonary fibrosis depending on the isoeffective doses was carried out. 




(a)



(b)

FIGURE 2. Appearance of patient with local breast cancer recurrence before (a) and after (b) neutron therapy (complete regression)

After the neutron therapy, 3 (11.5\%) out of 26 patients with recurrent breast cancer developed grade 1 radiation pulmonary fibrosis (RTOG/EORTC). Factors: strictly perpendicular direction of the cyclotron collimator to the chest wall, more than $148 \mathrm{~cm}^{2}$ irradiation area, 1.76 Gy single dose and more than 5 treatment sessions. Photon equivalent dose (PED) to the lung was $33 \mathrm{~Gy}$. In the case of mixed neutron-photon radiation therapy, 11 (25\%) out of the 44 patients with local recurrence developed radiation-induced pulmonary fibrosis (grade 1 pulmonary fibrosis in $4(9 \%)$ patients, grade 2 in $6(14 \%)$ patients and grade 3 in $1(2 \%)$ patients).

In Group II patients with unfavorable prognostic factors, moist desquamation after the neutron therapy was observed only in $3(5 \%)$ out of the 65 patients, requiring administration of magneto-laser therapy. Radiation-induced pulmonary fibrosis was diagnosed in only $4(6 \%)$ out of the 65 patients with breast cancer. Radiation-induced damages to lung tissue were treated with antibacterial and hormonal agents with a positive effect. The 7-year recurrence-free survival rate in the study group of the patients receiving neutron therapy to the region of the anterior chest wall was $93.4 \pm 4.6 \%$. In the control group of the patients who received electron beam therapy, the corresponding value was $77.5 \pm 7.9 \%(p=0.04)$. The overall 7 -year survival rate was $85.4 \pm 5.3 \%$ in the study group and $43.3 \pm 9.2 \%$ in the control group $(p=0.0002)$.

A significant statistical difference between the groups was not found; however, the following facts should be given in favor of the neutron therapy, namely: 1) combination of several unfavorable prognostic factors in breast cancer patients in the study group, 2) development of radioresistant forms of local recurrences after electron beam therapy in the control group.

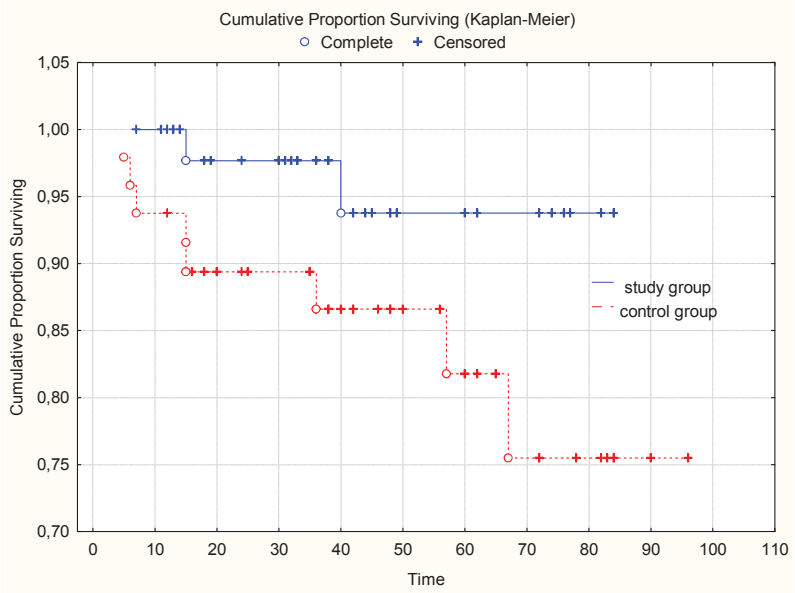

FIGURE 3. The 7-year recurrence-free survival rate in the study and control groups 


\section{CONCLUSIONS}

The neutron therapy was shown to be effective in multimodality treatment of patients with locally advanced breast cancer, and it is often the only treatment to prolong the life of patients with locally advanced breast cancer recurrence.

\section{ACKNOWLEDGMENTS}

The study reported in this article was conducted according to accepted ethical guidelines involving research in humans and/or animals and was approved by an Ethical Committee of Tomsk Cancer Research Institute.

The study is compliant with the ethical standards as currently outlined in the Declaration of Helsinki.

All individual participants discussed in this study, or for whom any identifying information or image has been presented, have freely given their informed written consent for such information and/or image to be included in the published article.

The study was financially supported by the Fundamental Research Program of the Russian Academy of Sciences, project No. 093.

\section{REFERENCES}

1. S. V. Kanaev, The role of radiotherapy in breast cancer treatment, Practical Oncology 1, 45-51 (2002).

2. V. F. Semiglazov and V. V. Semiglazov, Adjuvant chemoradiation therapy for breast cancer, Practical Oncology 9(1), 9-15 (2008).

3. E. G. Tyrsina, Study of the phenomenon of acquired radioresistance of tumor cells, Ph.D. thesis, Moscow, 2002.

4. I. A. Gulidov, Ju. S. Mardynskij, A. F. Cyb, and A. S. Sysoev, Neutrons of nuclear reactors in the treatment of malignant neoplasms, Digest of Conference Materials (Obninsk, 2001).

5. O. V. Gribova, Results of combined modality treatment and radiation therapy using $6.3 \mathrm{MeV}$ fast neutrons for salivary gland cancer and prognosticly unfavorable thyroid gland cancer, Siberian J. Oncol. 1, 51-52 (2009).

6. V. V. Velikaya, L. I. Musabaeva, Zh. A. Startseva, and V. A. Lisin, Fast neutrons of $6.3 \mathrm{MeV}$ in complex treatment of patients with breast cancer local recurrences, Voprosy Oncologii 61(4), 583-585 (2015).

7. S. G. Stuchebrov, I. A. Miloichikova, and I. B. Danilova, Measurement technique of dose rate distribution of ionization sources with unstable in time beam parameters, J. Phys. Conf. Ser. 671, 012057-1-5 (2016).

8. S. G. Stuchebrov, I. A. Miloichikova, and I. B. Danilova, The X-ray beam passage through the collimator made of different materials: numerical simulation, J. Phys. Conf. Ser. 671, 012012-1-5 (2016).

9. S. G. Stuchebrov, I. A. Miloichikova, A. L. Melnikov, and M. A. Pereverzeva, Numerical simulation of the microtron electron beam absorption by the modified ABS-plastic, J. Phys. Conf. Ser. 671, 012036-1-5 (2016).

10. S. G. Stuchebrov, I. A. Miloichikova, and K. O. Shilova, The dosimetric parameters investigation of the pulsed X-ray and gamma radiation sources, J. Phys. Conf. Ser. 671, 012051-1-6 (2016).

11. I. A. Miloichikova, S.G. Stuchebrov, G. K. Zhaksybaeva, and A. R. Vagner, Application of traditional and nanostructure materials for medical electron beams collimation: numerical simulation, IOP Conf. Ser. Mater. Sci. Eng. 98, 012011-1-6 (2015).

12. V. A. Lisin, TDF model for teletherapy of malignant neoplasms by fast neutrons, Med. Radiology 9, 9-12 (1988).

13. V. A. Lisin, Dosimetry computer therapy planning of malignant neoplasms by fast neutron beam of U-120 cyclotron, Medical Radiology 1, 26-28 (1991).

14. V. A. Lisin, V. V. Velikaya, and I. A. Miloichikova, Adaptation of linear-quadratic model for planning neutron therapy regimens, Siberian J. Oncol. 1, 33-37 (2015).

15. L. I. Musabaeva, Zh. A. Zhogina, V. V. Velikaya, and V. A. Lisin, Method of local breast cancer recurrence treatment, RU Patent, No. 2286818 (2006).

16. L. I. Musabaeva, V. V. Velikaya, Zh. A. Startseva, and V. A. Lisin, Method of preventing local recurrence in patients with primary locally advanced breast cancer (T2-4N0-3M0-1), RU Patent, No. 2444386 (2012). 